\title{
ACTE, PUISSANCE ET VIRTUALITÉ UNE GÉNÉALOGIE
}

\author{
Charles Alunni et Pierre CAYe
}

Quelle que soit la puissance d'intuition à l'origine du couple acte/ puissance, son fonctionnement, tel qu'Aristote l'aura modélisé, apparaît à maints égards aporétique, incapable surtout de rendre compte de la question même pour laquelle Aristote l'avait forgé : le mouvement. Giovanni Gentile ${ }^{1}$ reprochera en particulier à Aristote, dans L'Esprit, acte pur, de manquer totalement la question du processus d'actuation, c'est-à-dire de l'essence du mouvement "en-train-de-se-faire ": «La puissance, la matière, écrit-il, c'est du pas encore, tandis que la forme et l'acte c'est du déjà fait, du déjà épuisé ${ }^{2}$.» Bref, dans l'un et l'autre cas, du non-être. «De sorte, précise Gentile, qu'en analysant l'individu, on se trouvera inévitablement devant les deux éléments qui le constituent, sans pouvoir en saisir le rapport qui est précisément le processus d'actuation de l'individu. »

Cette aporie, loin d'invalider ces notions d'acte et de puissance, va les porter au premier plan et susciter de nouvelles configurations bien plus

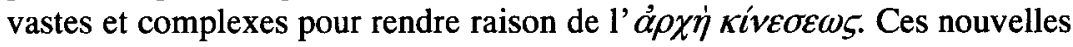
configurations brisent l'équilibre qu'Aristote tentait de maintenir entre les deux termes, et reconstituent leur dispositif en privilégiant tantôt la puissance, selon la doctrine de la toute-puissance ${ }^{3}$, tantôt l'acte, selon la doctrine de l'acte pur ${ }^{4}$.

1. Giovanni Gentile (1875-1944), sans doute le philosophe italien le plus imposant du $\mathrm{xx}^{\mathrm{e}}$ siècle, est le fondateur de l'actualisme ou philosophie de l'acte pur. On essaiera de montrer en quoi le fait que cet alter ego de Martin Heidegger ait intitulé sa propre philosophie "philosophie de l'acte pur " est hautement emblématique d'une configuration renouvelée du dispositif " acte, puissance, virtualité " dès l'ouverture du $\mathrm{xx}^{\circ}$ siècle. Il aura, en effet, fallu attendre ce siècle pour qu'un corpus philosophique donne son nom doctrinal à cette catégorie fondamentale.

2. G. GenTtLe, L'Esprit, acte pur, trad. franç. Aline Lion, Paris, Alcan, 1925, p. 57.

3. De Plotin qui l'aura mise en place, à Heidegger qui interroge cette notion de $\delta v v a \mu \zeta$ dans son cours du semestre d'été 1931 donné à Fribourg sous le titre, Aristote, Métaphysique $\theta$ 1-3. De l'essence et de la réalité de la force, trad. franç. Bernard STEvens et Pol VANDEVELDE, Paris, Gallimard, 1991.

4. Qui emporte avec elle la notion d'

Revue de synthèse : $4^{e}$ S. $n^{0}$ 1, janv.-mars 1999, p. 17-19. 
La toute-puissance n'est pas une doctrine du pouvoir absolu, mais de l'omnipossibilité : tout-puissant ne veut pas dire à l'origine que l'on peut tout, mais que tout est possible. Or, ici, le possible en tant qu'il est possible est déjà existence. « Des milliers de combinaisons chimiques, écrit Oswald Spengler, ne se produisent jamais et ne seront jamais réalisées, mais elles seront démontrées comme possibles, et par conséquent elles existent ${ }^{5}$." Dans ce cadre, l'acte compte peu : il ne s'agit plus de passer de la puissance à l'acte, mais de garder l'acte dans un état de diminution qui permette précisément de laisser ouvertes les substances à la variabilité de leurs mouvements. Dans ces conditions, ce qui fait que le possible existe et tient n'est plus l'acte, mais la mise en relation des possibles entre eux. In fine, c'est la mise en relation qui détermine la possibilité. L'omnipossibilité fonde les pensées du système, du réseau, du rhyzome. Instituée en système ou en réseau, l'omnipossibilité est alors amenée à faire l'épreuve de l'entropie, de la déperdition de pouvoir qu'implique tout déploiement relationnel. C'est pourquoi l'omnipossibilité est condamnée à en appeler au fantasme de la toute-puissance du principe pour justifier la cohésion et son maintien. Le dispositif dès lors oscille nécessairement entre immanence de l'omnipossibilité et transcendance de la toute-puissance. Ce point d'opposition (et de renvoi spéculaire) transcendance/immanence, récurrent tout au long du $\mathrm{xx}^{\mathrm{e}}$ siècle, est déjà inscrit dans l'ordre du parasitage théologique. À l'époque «moderne », il recouvre essentiellement le clivage cartésien/ spinoziste. Il reste dès lors principiellement en deçà du couple acte/ puissance : il ne change rien à la configuration métaphysique elle-même.

De l'omnipossibilité à la toute-puissance, le dispositif se durcit et devient contradictoire : il impose la nécessité de la toute-puissance théologique ou cosmique à ce qui n'était que le jeu libre et infini des variations de l'Être. Le fatum de la cosmologie stö̈cienne fait retour. C'est pour répondre à ce défi d'un fatum sans destin, que le dispositif de l'acte et de la puissance prend une nouvelle configuration tout opposée: l'acte pur. L'acte pur est ce qui advient à l'Être à partir précisément de cette impuissance absolue et inconditionnée qui menace l'omnipossibilité, mais que l'omnipossibilité ne cesse de dénier sous le masque de la toute-puissance. L'acte pur naît au cœur même de l'impossibilité de l'omnipossibilité. Il libère l'Être de l'emprise de l'harmonie du système, du flux des réseaux, du lien substantiel du conamen universale. Mais cette libération ne signifie pas pour autant le retour à l'entéléchie apaisée de la substance aristotélicienne : elle, qui sous le régime de l'omnipossibilité subissait le travail constant des déformations morphogénétiques, expérimente, sous le régime de l'acte pur,

5. Oswald Spengler, Le Déclin de l'Occident, Paris, Gallimard, 1948, vol. I, chap. II, « Idée de destin et principe de causalité », p. 158. 
le travail de faille et de fragmentation sans recomposition que lui impose l'acte dans le mouvement inconditionné de son émergence. L'acte est un trait, une ligne de bris qui fuit sans jamais se connecter avec d'autres lignes, au risque de tourner en pulsion de mort et en destruction.

Ce dispositif spéculatif et praxéologique s'inscrit de façon généralisée au cœur du $\mathrm{xx}^{\mathrm{e}}$ siècle. Mais c'est, dès lors, pour aller jusqu'au bout de ses configurations, de leurs apories, de leurs contradictions. Loin de se défaire, la métaphysique a plutôt cherché, sous les figures aussi diverses de « l'acte ou [du] vouloir inactuels ${ }^{6} »$ (Husserl), de «l'acte libre comme puissance créatrice de la durée ${ }^{7}$ » (Bergson), de l'Ereignis (Heidegger), de la « souveraineté $^{8}$ " (Bataille) ou de la «potentialité réelle ${ }^{9}$ " (Schmitt), à en proposer une nouvelle synthèse qui prenne en compte l'ensemble des expériences propres à chaque nouvelle configuration. De surcroît, elle a recentré la question ontologique qui, chez Aristote, restait éclatée entre l'intuition catégoriale, le jugement vrai, la métaphysique de l'être (substance et accident) et celle du mouvement (acte/puissance), autour de ce dernier couple appelé à retraduire dans sa propre logique les trois autres approches de l'Être.

Charles Alunni et Pierre Caye (1996).

6. Edmund HUSSERL, Idées directrices pour une phénoménologie et une philosophie phénoménologique pures. T. I : Introduction générale à la phénoménologie pure, trad. de Paul RICEUR, Paris, Gallimard, 1950, \$\$ 35, 37, 84 ad finem et 115.

7. Henri Bergson, Essai sur les données immédiates de la conscience, in Euvres, Paris, Presses universitaires de France, 1959. Voir, en part., sur son questionnement du dispositif acte/puissance, sa thèse complémentaire intitulée Quid de loco Aristoteles senserit (1889). Voir, également, Louis de Broglie, Olivier Costa de Beauregard et al., Bergson and the evolution of physics, dir. P. A. Y. Gunter, Knoxville, TN, University of Tennessee Press, 1969.

8. Georges Bataille, Euvres completes, Paris, Gallimard, 1976, vol. VI et VIII.

9. Carl Schmitr, La Notion du Politique, 1932, trad. franç. de Julien Freund, Paris, Calmann-Lévy, 1972. 